\title{
The vital role of physiotherapy during COVID-19: A systematic review
}

\author{
P. Antony Leo Asser ${ }^{1}$ and K. Soundararajan ${ }^{2, *}$ \\ Faculty of Physiotherapy, Sri Ramachandra Institute of Higher Education and Research \\ (Deemed to be University), Chennai, India
}

Received 16 March 2021

Accepted 14 September 2021

\begin{abstract}
.
BACKGROUND: The current COVID-19 pandemic has changed the entire world population's physical and mental wellbeing irrespective of the person being infected or not. Flourishing numbers of new research recommends physiotherapy for the management of COVID-19 patients. However, there are cavities in the study in the recommendation of physiotherapy specific to the current pandemic.

OBJECTIVE: This review aimed to synthesize physiotherapy-related articles to COVID-19 and summarize their efficacious highlights.

METHODS: For the literature search PubMed, PEDro, DOAJ and The Cochrane Database of Systematic Reviews were used. The keywords included "Physiotherapy", "COVID-19", and "Coronavirus". The Boolean search was applied as required. Selection criteria included studies that included physiotherapy intervention as a tool for recovery of COVID-19. Exclusion criteria included animal studies, non-COVID-19 studies and physiotherapy as an adjunct treatment. The study evaluated evidence of all full-text articles in English from December 2019 to August 2020.

RESULTS: Of the retrieved 577 articles, 390 articles were excluded at the title and abstract screening. 167 articles underwent full-text screening and further narrowed to 11 studies matching the expected criteria. 156 studies were excluded for various reasons.

CONCLUSION: The current study findings support that physiotherapy interventions facilitate recovery in COVID-19 patients and act as a protective barrier. Further results include a reduced length of stay in intensive care and reduced treatment cost since this outbreak has brought a significant economic burden to many countries.
\end{abstract}

Keywords: Coronavirus, health, physiotherapy, COVID-19

\footnotetext{
${ }^{1}$ ORCID: https://orcid.org/0000-0002-8489-7243.

${ }^{2}$ ORCID: https://orcid.org/0000-0002-3711-8702.

*Address for correspondence: Mr. K. Soundararajan, MPT, Postgraduate, Faculty of Physiotherapy, Sri Ramachandra Institute of Higher Education and Research (Deemed to be University), Chennai, India. E-mail: k.soundararajan1995@gmail.com.
}

\section{Introduction}

The current Coronavirus Disease 2019 (COVID19) pandemic has changed the entire world population's physical and mental wellbeing irrespective of the person being infected or not. The COVID19 is a highly ingenious disease caused by a new coronavirus, namely severe acute respiratory 
syndrome coronavirus-2 (SARS-CoV-2). In China, Wuhan city of Hubei province has reported a cluster of pneumonia cases to the World Health Organization (WHO) at the end of December 2019. The WHO declared COVID-19 a pandemic on March 11, 2020 $[1,2,16]$.

Angiotensin-converting enzyme 2 (ACE2) is customarily attached to type I and II alveolar cells. ACE2 helps in secreting surfactant, transepithelial movement of water, alveolar epithelium regeneration, and xenobiotic metabolism. When the virus invades the lung tissue, it damages the alveolar cells, leading to acute respiratory distress syndrome (ARDS), sepsis, septic shock, kidney failure, and cardiac failure, eventually resulting in death [3]. COVID19 infected individual's usually demonstrated fever, cough, fatigue, sputum production, and shortness of breath. $80 \%$ of cases were asymptomatic without any symptoms; $15 \%$ of patients require oxygen support in the hospital, and $5 \%$ of cases are critically ill, requiring mechanical ventilation and life support in the intensive care unit (ICU) [2, 4].

A physiotherapist is a multi-professional team member and plays an essential role in providing ventilator support and rendering respiratory techniques to critically ill patients. Moreover, the rehabilitation intervention improves individuals' quality of life and functional abilities with deficits [5]. In particular, cardio-respiratory physiotherapy aid in the clearance of secretions in individuals who cannot clear independently with or without associated comorbidities leading to favourable recovery. COVID-19 patients in ICU are managed by prolonged protective ventilation, sedation prone to ICU-acquired weakness (ICU-AW), consequently impacting the morbidity and mortality rate. So, it was mandatory to provide early rehabilitation for all COVID-19 cases to enhance the rapid functional recovery rate $[6,7]$. Flourishing numbers of new research recommends physiotherapy for the management of COVID-19 patients. However, there are lacunae in research in the recommendation of physiotherapy specific to the current pandemic. This review aimed to synthesize physiotherapy related to COVID-19 studies and summarize their efficacious highlights. The overall aim of this systematic review is to reconnaissance the current shreds of evidence pertaining to physiotherapy interventions in COVID-19. The distinct objectives were to compile the prevailing evidence explicitly related to physiotherapy interventions for people with COVID-19 and pinpoint the deficit in evidence that may require further studies.

\section{Methods}

\subsection{Study setting}

A systematic review was conducted in the following defined format and the study was registered in the open science framework (DOI 10.17605/ OSF.IO/EDR6M).

\subsubsection{Identification of research question}

The PICOT(S) method proposed by Sackett et al. [7] was used in this systematic review to formulate a search strategy.

P (Population) - people infected by COVID-19.

I (Intervention) - physiotherapy intervention includes any form of exercise or physical activity carried out within the physiotherapist's scope.

C (Comparator) - any comparator; no comparator.

$\mathbf{O}$ (Outcomes) - any physical or self-reported outcome tool which measures subjectively or objectively determine whether the patient has demonstrated the change in COVID-19 subjects.

T (Time) - all studies from December 2019 to August 2020 were considered but were not limited according to follow-up time.

S (Study design) - any study design considered excluding articles published in the protocol stage.

\subsubsection{Identify the relevant studies}

A comprehensive literature search was conducted in the following databases: PubMed, PEDro, DOAJ and The Cochrane Database of Systematic Reviews. The keywords include "Physiotherapy", "COVID19", and "Coronavirus". The Boolean search was applied as required. Grey literature or hand searching literature was also considered in the scoping review process.

\subsubsection{Study selection}

After completing database searches, the collected citations were entered into Mendeley Desktop version 1.19.4 for checking duplicate citations. In stage one, all article titles and abstracts were screened independently by two reviewers. Stage two involves full-text evaluation, and further articles were segregated to fulfill the study objectives. A third expert reviewer cross-checked and finalized the consensus and consistency of studies included in the scoping review. Manual hand searching was performed to ensure comprehensiveness.

A data extraction table was tabulated to collect data from selected studies, including the authors with 


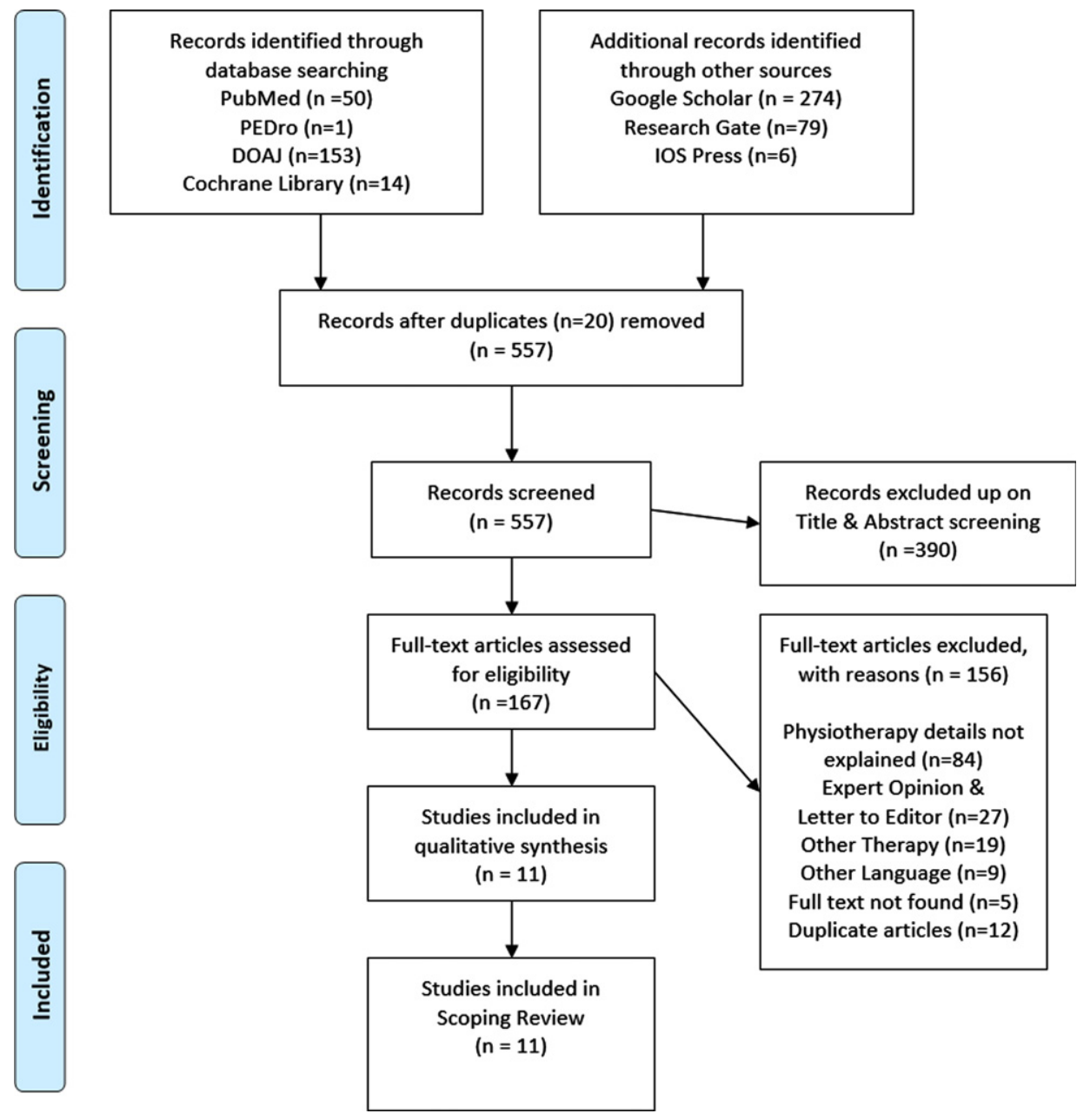

Fig. 1. Flowchart of the study selection process.

year, country, samples, intervention, study design, and highlights. The data entries were cross verified by the reviewers to confirm accuracy. Finally, the data table was scrutinized by an expert reviewer.

A descriptive synthesis of collected articles was carried out after the data extraction procedure. Overall, this needs to narrate the available evidence and identify lacunae in the current literature.

\section{Results}

The characteristics of the included studies in this scoping review are shown in Fig. 1. Following study selection, 11 papers were included in the scoping review: Ten reviews and one survey analysis. The 11 most relevant studies are described in Table 1. Based on the reviews, we developed an algorithm to categorize COVID-19 patients on different variables (Fig. 2).

\section{Discussion}

A classification category was assigned for each COVID-19 patient by the physiotherapist referred for rehabilitation. This classification system is based on various study suggestions that got extracted from the scoping review. The purpose of the algorithm was to standardize rehabilitation protocol for COVID-19 patients worldwide and this was the first systematic review intended to classify and frame an algorithm.

This systematic review aimed to determine the current literature regarding rehabilitation of people with COVID-19 and identify gaps in the evidence. Based on the purpose of the study, the broad inclusion and 
Table 1

Description of the 11 studies in this systematic review

\begin{tabular}{lcll}
\hline Authors & Location & Design & Intervention \\
\hline Sheehy et al. [21] & Canada & Review & $\begin{array}{c}\text { Respiratory, mobility and } \\
\text { functional rehabilitation }\end{array}$ \\
& & \\
Lazzeri et al. [22] & Italy & Review & $\begin{array}{l}\text { Respiratory Physiotherapy (PT) } \\
\text { recommendation for ventilated } \\
\text { patients }\end{array}$ \\
& &
\end{tabular}

Thomas et al. [9] Australia Review PT workforce planning, preparation, selection of physiotherapy treatment and Personal Protective Equipment (PPE).

Wei et al. [23]

China Survey analysis Perform more physical exercises and exercises at home

Mohamed et al. [24] Turkey Review Aerobic exercises

Righetti et al. [10] Brazil Review Conventional, chest physiotherapy, exercise and early mobilization

Negrini et al. [25] Italy Review Prone position during ventilation; Passive mobilization

Li et al. [26]

China Review

A new era of smart remote rehabilitation approaches which includes wearable devices, mobile phone apps and virtual reality

Vitacca et al. [27] Italy $\quad$ Review $\quad$ Respiratory rehabilitation and physiotherapy interventions

Paneroni et al. [28] Italy Review

Posture, reconditioning with leg/arm cranking and exercises, assessment by short-physical performance battery (SPPB) and 1-minute sit-to-stand test (1-STS)

Vitacca et al. [29] Italy Review

Pulmonary rehabilitation in COVID-19 patients recovering from acute respiratory failure

Highlights

Rehabilitation after COVID-19 includes proper assessment; individualized progressive treatment plan which focuses on function and disability will help each patient to maximize their function and quality of life.

A quick respiratory physiotherapist reference guide to set up treatments for the management in acute stages of patients suffering from COVID-19.

Clinical Practise recommendation for physiotherapists in acute care management for adult patients with confirmed or suspected COVID-19.

Physical exercise with suitable intensity and quantity can relieve psychological pressure, eliminate mental tension, and achieve the effect of psychological stability.

The review summarizes that increasing the aerobic capacity is recommended because it has the potential of improving immune and respiratory functions which would help to counter COVID-19.

Physiotherapy plays a fundamental role throughout patient hospitalization. However, the hospital physiotherapy team must be well oriented towards specific care to reduce infection risk and to provide the best patient care.

Early rehabilitation should be granted to inpatients with COVID-19; tele-rehabilitation may represent the first option for people at home.

Rehabilitation emphasizes treatment with appropriate methods at the right time, including bed positioning, respiratory training, exercises and mobilization, physical modalities, occupational therapy, speech and swallowing therapy.

For COVID-19 patients in isolation, rehabilitation programs can conduct remotely by a tele-health system (Educational videos, tele-consultation, webcams, etc.).

Following patient discharge, patients should receive indications to cope with physical activity regarding functions, capacity, and participation when the patient is cured with no longer a risk of contagion.

As main topics, respiratory physical therapists have been involved in oxygen therapy management in almost a third of the admitted patients, reconditioning exercises in $60 \%$ of the cases, and initial and final functional motor capacity assessment in all COVID-19 patients.

COVID-19 patients with primarily respiratory conditions, pulmonary rehabilitation has an important role to play in promoting recovery and improving community reintegration by increasing mobility, autonomy and health-related quality of life. 


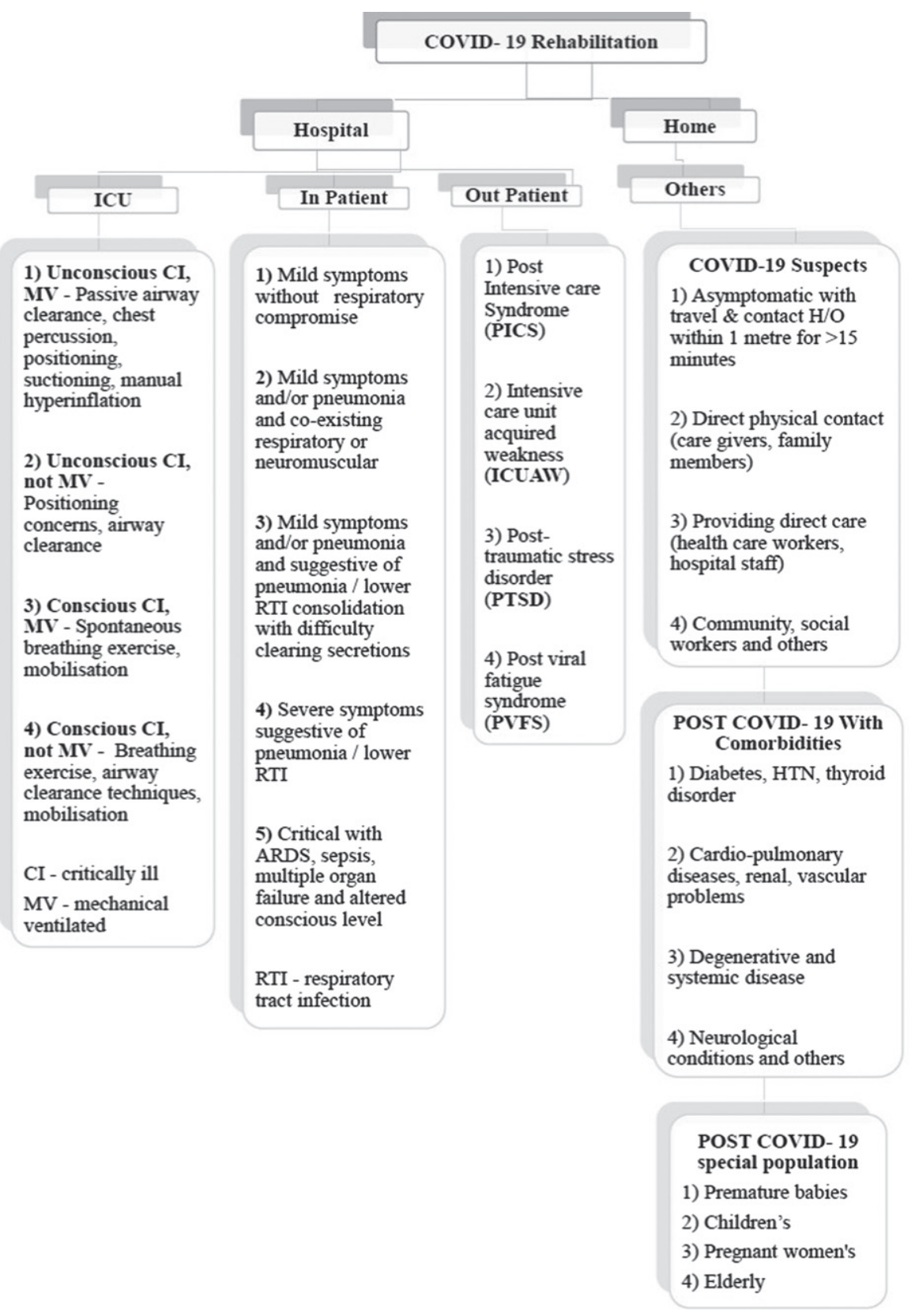

Fig. 2. COVID-19 rehabilitation-based classification system for COVID-19 patients.

exclusion criteria were framed. A total of 11 articles that met the inclusion criteria were considered for this review. The review demonstrated a lack of physiotherapy research for COVID-19 patients who were both symptomatic and asymptomatic undergoing rehabilitation. Very few studies discussed physiotherapy intervention in a detailed manner, even though the majority of reviews failed to describe the physiotherapy component at all.
Few reviews related to search strategies were found, but only a few explicitly focused on physiotherapy intervention in detail. Similarly, the majority of the reviews included studies with physiotherapy as part of a rehabilitation process. The few retrieved RCTs were found to represent the protocols and hence it was not considered. No observational studies, experimental studies, clinical trials, systematic review and meta-analysis studies were found during 
the data extraction, which included physiotherapy interventions for COVID-19 patients exclusively. Most of the review articles are statements from various physiotherapy associations of different countries, council recommendations, and consensus statements. There was no contrasting evidence reported regarding physiotherapy being contraindicated for COVID-19 patients. Only a few physiotherapy techniques (abdominal breathing exercise, incentive spirometry, pursed-lip breathing exercise, manual therapy/stretches for ribcage, exercise training and mobilization during clinical instability) were contraindicated in the acute phase of COVID-19 patients [8].

Individuals infected with COVID-19 require hospital admission and can be further categorized into patients admitted in the intensive care unit (ICU) or inward patient ward. Patients in ICU care can be further sub-grouped based on the level of consciousness and with or without mechanical ventilator support. Based on sub-grouping, the physiotherapy intervention shown in Fig. 2 can be administrated. Patients admitted inward at the hospital can be further subgrouped, screened and treated according to Thomas et al.'s recommended framework [9]. Physiotherapy interventions for each group must be started once a referral was sought.

Prone positioning for $12-16$ hours is recommended for all ventilator support patients. The advantage of the prone position is that it relieves severe hypoxemia and prevents ventilator-induced lung injury. When compared to supine positioning, the prone position promotes more alveolar recruitment and reduces overinflated lung areas. In the prone position, transpulmonary pressure along the posterior to the anterior axis is more homogeneously distributed than in the supine position. So prone positioning was suggested to contribute to a decrease in lung stress and strain. Additionally, the physiotherapist must be expertise in safely performing prone positioning of ventilated patients to avoid complications, including airway and pressure area issues [10-12].

Following hospital stay (ICU or in-patient ward) [17], COVID-19 survivors may experience new or worsening complications such as post-intensive care syndrome (PICS), intensive care unit acquired weakness (ICUAW), post-traumatic stress disorder (PTSD), post-viral fatigue syndrome (PVFS), critical illness polyneuropathy (CIP) and critical illness myopathy (CIM) [13]. These complications could be benefited from outpatient physiotherapy services based on scheduled appointments to avoid overcrowding and congestion and further shifted from rehabilitation to telerehabilitation.

Telerehabilitation demonstrated a viable and effective alternative rehabilitation method for patients who are unable to access in-person physiotherapy services in the outpatient department. A well-structured and defined telerehabilitation service in this current COVID-19 pandemic helps COVID-19 patients who are in-home quarantine, community and other patients who are seeking physiotherapy treatment [14]. Telerehabilitation is set to bridge the gap in physiotherapy access during the time of lockdown when social distancing is mandated. Telerehabilitation reduces the chance of spread and transmission of COVID-19, PPE usage cost, and saving traveling time [18-20]. Telerehabilitation is not standard care of practice for rehabilitation and is yet not covered by insurance companies. Changes in insurance rules for claiming telehealth services probably help patients improve their quality and functional abilities and decrease insurance company expenditure [15].

People (post-COVID-19 recovered, COVID-19 contacts/suspects) quarantined at home with or without comorbidities due to restricted mobility have the risk of frailty, sarcopenia, dementia, psychological and musculoskeletal issues. So, it was advisable to perform a multi-component rehabilitation program to maintain and improve the health status of all individuals [16]. People at home quarantine could be further sub-grouped into COVID-19 suspects, post-COVID-19 with comorbidities, post-COVID-19 special population as represented in Fig. 2. Physiotherapy interventions for each patient belonging to each subgroup may be tailored-made/individualized or standard exercise programs focused on the patient's current and anticipated problem list. These exercise programs must fulfill the recommendations of clinical practice.

COVID-19 rehabilitation can be based on only generalized experience, recommendations and expert opinions. Even though exercise programs were recommended for COVID-19, all shreds of evidence lack involving COVID-19 patients. All researches are based on experience, recommendations/guidelines from associations/councils; hence, no judgment can be made from these features. Since the amount of the data was reviews, additional analysis was precluded. The study's limitations were search databases, review articles and period. The literature that was included in the systematic review was not reviewed critically. Future research is assured to know the effectiveness of physiotherapy in COVID-19 patients, including 
all databases for literature search and validating the proposed algorithm with less risk of bias.

\section{Conclusions}

The current systematic review findings support the use of physiotherapy interventions aids in quicker recovery of COVID-19 patients and act as a protective barrier and boost immune and respiratory system. Early rehabilitation reduces the length of stay in the ICU/hospitals and further decreases the treatment cost of COVID-19.

\section{Acknowledgments}

The authors gratefully acknowledge the Faculty of Physiotherapy, Sri Ramachandra Institute of Higher Education and Research, Chennai.

\section{Conflict of interest}

The authors declare no potential conflicts of interest concerning the research, authorship, and/or publication of this article.

\section{Funding}

The authors received no financial support for this article.

\section{References}

[1] Del Rio C, Malani PN. 2019 novel coronavirus important information for clinicians. Jama. 2020;323(11):1039-40.

[2] Covid CD, Team R, Bialek S, Boundy E, Bowen V, Chow N, Cohn A. Severe outcomes among patients with coronavirus disease 2019 (COVID-19)—United States, February 12-March 16, 2020. Morbidity and Mortality Weekly Report. 2020;69(12):343.

[3] Abdullahi A. Safety and efficacy of chest physiotherapy in patients with COVID-19: a critical review. Frontiers in Medicine. 2020;7:454.

[4] Guan WJ, Ni ZY, Hu Y, Liang WH, Ou CQ, He JX, Liu L, Shan H, Lei CL, Hui DS, Du B. Clinical characteristics of coronavirus disease 2019 in China. New England Journal of Medicine. 2020;382(18):1708-20.

[5] Goñi-Viguria R, Yoldi-Arzoz E, Casajús-Sola L, AquerretaLarraya T, Fernández-Sangil P, Guzmán-Unamuno E, Moyano-Berardo BM. Respiratory physiotherapy in intensive care unit: Bibliographic review. Enfermería Intensiva (English ed.). 2018;29(4):168-81.
[6] Xie J, Tong Z, Guan X, Du B, Qiu H, Slutsky AS. Critical care crisis and some recommendations during the COVID19 epidemic in China. Intensive Care Medicine. 2020;46(5): 837-40.

[7] Sackett DL, Straus, MD, Richardson, WS, Rosenbergy, W, Haynes, RB. Evidence-Based Medicine: How to Practice and Teach EBM London, UK: Churchill Livingstone; 2000.

[8] Shamsi S, Al-Shehri A, Khan S, Al Torairi N, Al Amoudi KO. Importance of Physiotherapy in COVID-19: A Recommendation. Int J Rec Innov Med Clin Res. 2020;2(3):46-54.

[9] Thomas P, Baldwin C, Bissett B, Boden I, Gosselink R, Granger CL, Hodgson C, Jones AY, Kho ME, Moses R, Ntoumenopoulos G. Physiotherapy management for COVID-19 in the acute hospital setting: clinical practice recommendations. Journal of Physiotherapy. 2020;66(2): 73-82.

[10] Righetti RF, Onoue MA, Politi FV, Teixeira DT, Souza PN, Kondo CS, Moderno EV, Moraes IG, Maida AL, Pastore L, Silva FD. Physiotherapy care of patients with coronavirus disease 2019 (COVID-19)-a Brazilian experience. Clinics. 2020;75.

[11] Alhazzani W, Møller MH, Arabi YM, Loeb M, Gong MN, Fan E, Oczkowski S, Levy MM, Derde L, Dzierba A, Du B. Surviving Sepsis Campaign: guidelines on the management of critically ill adults with Coronavirus Disease 2019 (COVID-19). Intensive Care Medicine. 2020;46(5):854-87.

[12] World Health Organization. Clinical management of severe acute respiratory infection ( SARI) when COVID-19 disease is suspected: interim guidance, March 13 2020. World Health Organization; 2020.

[13] Connolly B, O'neill B, Salisbury L, Blackwood B. Physical rehabilitation interventions for adult patients during critical illness: an overview of systematic reviews. Thorax. 2016;71(10):881-90.

[14] Cottrell MA, Russell TG. Telehealth for musculoskeletal physiotherapy. Musculoskeletal Science and Practice. 2020;48:102193.

[15] Bettger JP, Thoumi A, Marquevich V, De Groote W, Battistella LR, Imamura M, Ramos VD, Wang N, Dreinhoefer KE, Mangar A, Ghandi DB. COVID-19: maintaining essential rehabilitation services across the care continuum. BMJ Global Health. 2020;5(5):e002670.

[16] Siddiqui AA, Alshammary F, Amin J, Rathore HA, Hassan I, Ilyas M, Khursheed Alam M. Knowledge and practice regarding prevention of COVID-19 among the Saudi Arabian population. Work. 2020 January 1 (Preprint):1-9.

[17] McKee G, Cronin C, Vasquez P, Burke D, O'Flaherty D. The physiotherapy experience of managing patients with COVID-19. Physiotherapy Practice and Research. 2020; 41(2):95-7.

[18] Jesus TS, Landry MD, Jacobs K. A 'new normal'following COVID-19 and the economic crisis: Using systems thinking to identify challenges and opportunities in disability, telework, and rehabilitation. Work. 2020 January 1 (Preprint):1-0.

[19] Capozzo R, Zoccolella S, Frisullo ME, Barone R, Dell'Abate MT, Barulli MR, Musio M, Accogli M, Logroscino G. Telemedicine for delivery of Care in Frontotemporal Lobar Degeneration during COVID-19 pandemic: results from southern Italy. Journal of Alzheimer's Disease. 2020 July 21 (Preprint):1-9.

[20] Rabatin AE, Lynch ME, Severson MC, Brandenburg JE, Driscoll SW. Pediatric telerehabilitation medicine: Making your virtual visits efficient, effective and fun. Journal of Pediatric Rehabilitation Medicine. 2020 January 1 (Preprint):1-6. 
[21] Sheehy LM. Considerations for post acute rehabilitation for survivors of COVID-19. JMIR Public Health and Surveillance. 2020;6(2):e19462.

[22] Lazzeri M, Lanza A, Bellini R, Bellofiore A, Cecchetto S, Colombo A, D'Abrosca F, Del Monaco C, Gaudiello G, Paneroni M, Privitera E. Respiratory physiotherapy in patients with COVID-19 infection in acute setting: a Position Paper of the Italian Association of Respiratory Physiotherapists (ARIR). Monaldi Archives for Chest Disease. 2020;90(1).

[23] Wu K, Wei X. Analysis of psychological and sleep status and exercise rehabilitation of front-line clinical staff in the fight against COVID-19 in China. Medical Science Monitor Basic Research. 2020;26:e924085-1.

[24] Mohamed AA, Alawna M. Role of increasing the aerobic capacity on improving the function of immune and respiratory systems in patients with coronavirus (COVID-19): A review. Diabetes \& Metabolic Syndrome: Clinical Research \& Reviews. 2020;14(4):489-96.

[25] Negrini S, Ceravolo MG, Côté P, Arienti C. A systematic review that is "rapid" and "living": a specific answer to the COVID-19 pandemic. Journal of Clinical Epidemiology. 2021.
[26] Li J. Rehabilitation management of patients with COVID19: lessons learned from the first experience in China. European Journal of Physical and Rehabilitation Medicine. 2020;56(3):335-8.

[27] Vitacca M, Carone M, Clini EM, Paneroni M, Lazzeri M, Lanza A, Privitera E, Pasqua F, Gigliotti F, Castellana G, Banfi P. Joint statement on the role of respiratory rehabilitation in the COVID-19 crisis: the Italian position paper. Respiration. 2020;99(6):493-9.

[28] Simonelli C, Paneroni M, Fokom AG, Saleri M, Speltoni I, Favero I, Garofali F, Scalvini S, Vitacca M. How the COVID-19 infection tsunami revolutionized the work of respiratory physiotherapists: an experience from Northern Italy. Monaldi Archives for Chest Disease. 2020;90(2).

[29] Vitacca M, Lazzeri M, Guffanti E, et al. Italian suggestions for pulmonary rehabilitation in COVID-19 patients recovering from acute respiratory failure: results of a Delphi process. Monaldi Arch Chest Dis. 2020;23(90):385-93. 